\title{
Effects of Methenamine Feeding Regime on Growth Performances, Gut Microbiota, Organs Histology and Haemato-Biochemical Profile of Broiler Chickens
}

\author{
Kengni Noubissie Guyssela Josiane ${ }^{1 *}$, Kana Jean Raphaël ${ }^{1}$, Ngouana Tadjong Ruben², \\ Yemdjie Mane Doriane Divine ${ }^{2}$, Ebile Dayan Agwa1, Tchouan Deffo Gilchrist ${ }^{1}$, \\ Necdem Tsafack Boris Valdes' ${ }^{1}$ Issa Bachar Issa1 \\ ${ }^{1}$ Laboratory of Animal Nutrition, Department of Animal Science, University of Dschang, Cameroon \\ ${ }^{2}$ Laboratory of Aquaculture and Demography of Fisheries Resources, Department of Aquaculture, institute of Fisheries and \\ Aquatic Science of Yabassi, University of Douala, Cameroon \\ Email: *kguysselajosiane@yahoo.fr
}

How to cite this paper: Josiane, K.N.G., Raphaël, K.J., Ruben, N.T., Divine, Y.M.D., Agwa, E.D., Gilchrist, T.D., Valdes, N.T.B. and Issa, I.B. (2021) Effects of Methenamine Feeding Regime on Growth Performances, Gut Microbiota, Organs Histology and Haemato-Biochemical Profile of Broiler Chickens. Open Journal of Animal Sciences, 11, 238-254.

https://doi.org/10.4236/ojas.2021.112019

Received: November 13, 2020

Accepted: April 23, 2021

Published: April 26, 2021

Copyright $\odot 2021$ by author(s) and Scientific Research Publishing Inc. This work is licensed under the Creative Commons Attribution International License (CC BY 4.0).

http://creativecommons.org/licenses/by/4.0/ (c) $\underset{\mathrm{By}}{\mathrm{By}}$ Open Access

\begin{abstract}
Microbial resistance in livestock has become a subject of great concern of public and scientific interest. This study was designed to assess the effects of methenamine feeding regime on growth performances of broilers chickens. For this purpose, 120 chicks of Cobb 500 strain, including 60 males and 60 females of 21 days old with an average weight of $639 \mathrm{~g}$ and $584 \mathrm{~g}$ respectively were used. They were randomly distributed in 60 experimental units of 2 chicks of same sex per cage until 49 days. Methenamine was incorporated in feed (TA), acidified (TEa) and non acidified (TE) water and compared to an antibiotic medicated diet as positive control $\left(\mathrm{T}^{+}\right)$and a ration without any supplement as negative control (T0). The main results showed that, regardless of the feeding regime, methenamine significantly $(\mathrm{p}<0.05)$ increased feed inteake, body weight, weight gain and decreased $(p<0.05)$ feed conversion ratio. Methenamine whatever the feeding regime induced a significant increase in lactic acid bacteria counts compared to coliforms and coccidies counts. Salmonella were absent throughout the trial period. Regardless of sex and feeding regime, hematological parameters were not significantly affected, with the exception of white blood cell and platelet concentration that decreased significantly $(\mathrm{p}<0.05)$ in male broilers. Serum content in ASAT (Aspartate-transferase), ALAT (Alanine-transferase), creatinine, urea and LDLcholesterol decreased significantly $(\mathrm{p}<0.05)$, while HDL-cholesterol increased. Histology of organs was not affected. Feeding methenamine to broiler chickens through drinking water can be used as an alternative to antibiotic
\end{abstract}


to improve growth performances.

\section{Keywords}

Antibiotic, Broiler Chickens, Gut Microbiota, Growth Performance,

Haemato-Biochemical Profile, Methenamine

\section{Introduction}

In order to solve the problem of bacterial resistance due to the use of antibiotics as feed additive, many compounds have been identified as an alternative to antibiotics growth promoters in poultry production. Among those compounds we can list phytobiotics [1], amino acid salts such as monosodium glutamate [2] and nitrogenous compounds such as methenamine [3].

Methenamine is an antimicrobial drug used in the treatment of urinary tract infections, prophylaxis and bacteriuria in humans [4] [5] [6]. It is relatively harmless and decomposes into formaldehyde and ammonia under acidic conditions or in the presence of proteins [7]. The bactericidal properties of formaldehyde fight against infection [8] [9]. In avian medicine, methenamine is often dispensed in the treatment of nephritis, pyelitis, pyelonephritis, ascites, edema and hepatitis [10]. It also has diuretic and anti-bleeding properties for the treatment of sulpha-drug intoxication, infectious bursal disease and coccidiosis [10]. It is used as a preservative to improve the silage process of feed for pigs, cattle, sheep, goats, rabbits and horses at a maximum rate of $600 \mathrm{mg} / \mathrm{kg}$ of fresh material [3] [11] and as cheese preservatives at a rate of $25 \mathrm{mg} / \mathrm{kg}$ [3] [7]. According to EFSA [12], the antimicrobial properties of formaldehyde have been widely exploited for the microbial decontamination of stored feeds, mainly fishmeal. At a dose of $660 \mathrm{mg}$ per kilogram of feed, formaldehyde is able to eliminate or reduce the number of pathogenic bacteria such as Escherichia coli and Salmonella typhimurium [11]. The high reactivity of formaldehyde with amine groups makes it possible to protect soybean, rapeseed and/or sunflower seed meal proteins from microbial degradation [11]. Based on the above properties, we believed that methenamine could be used to balance intestinal microbiota with positive consequences on growth performance in poultry.

The objective of this study was to evaluate the potential of methenamine in replacing the antibiotic growth promoters in poultry feed.

\section{Materials and Methods}

\subsection{Area of Study}

This study was carried out at the Teaching and Research Farm of the Faculty of Agronomy and Agricultural Sciences, University of Dschang, Cameroon. This farm is located at an altitude of $1420 \mathrm{~m}$ above sea level, between latitude $5^{\circ} 26^{\prime} \mathrm{N}$ and longitude $10^{\circ} 26^{\prime} \mathrm{E}$ with an equato-guinean climate, annual temperatures 
vary between $10^{\circ} \mathrm{C}$ and $25^{\circ} \mathrm{C}$. The annual rainfall ranges from 1500 to $2000 \mathrm{~mm}$, the wet season lasts from mid-March to mid-November and the dry season from late November to mid-March.

\subsection{Feed Additive and Experimental Birds}

Merck brand methenamine (Darmstadt, Germany) used in this study as a feed additive is in the form of white crystals and soluble in water.

A total of 120 three-week-old Cobb 500 strain broiler chicks were divided into 5 groups of 24 chicks each. Each group was subdivided into 12 replicates of 2 chicks per replicate. The chicks were housed in cages at a density of 1 chick/0.12 $\mathrm{m}^{2}$ up to 49 days. The chicks were given antistress once a week after handling. Feed and water were offered ad libitum.

\subsection{Dietary Treatments}

Dietary treatments consisted of supplementing basal control diet (T0) (Table 1) with $1 \mathrm{~g}$ of Doxcyclin/ $\mathrm{kg}$ of feed as a positive control $\left(\mathrm{T}^{+}\right), 1 \mathrm{~g}$ of methenamine/kg of feed (TA), $1 \mathrm{~g}$ of methenamine/L of drinking water (TE) and $1 \mathrm{~g}$ of methenamine/ $\mathrm{L}$ of drinking acidify water (TEa) obtained by adding $1 \mathrm{~g}$ of acetic acid per liter.

Table 1. Chemical composition of experimental diet.

\begin{tabular}{|c|c|}
\hline Ingredients & $\%$ \\
\hline Corn & 60.00 \\
\hline Wheat bran & 12.00 \\
\hline Cotton seed cake & 1.00 \\
\hline Fishmeal & 5.00 \\
\hline Soybean meal & 16.00 \\
\hline Shell & 1.00 \\
\hline Premix 5\%* & 5.00 \\
\hline Total & 100.00 \\
\hline \multicolumn{2}{|l|}{ Calculated chemical composition } \\
\hline Metabolic energy (kcal/kg) & 3000.90 \\
\hline Crude protein (\%) & 20.20 \\
\hline Energy/protein & 148.90 \\
\hline Calcium (\%) & 1.16 \\
\hline Phosphorus (\%) & 0.53 \\
\hline Calcium/Phosphorus & 2.18 \\
\hline Lysine (\%) & 1.20 \\
\hline Methionine (\%) & 0.44 \\
\hline Price of kg (FCFA) & 254.50 \\
\hline
\end{tabular}

${ }^{*}$ Premix 5\%: crude protein $=40 \%$, Lysine $=3.3 \%$, Methionine $=2.40 \%$, Calcium $=8 \%$, Phosphorus $=$ $2.05 \%$, metabolizable energy $=2078 \mathrm{kcal} / \mathrm{kg}$. 


\subsection{Data Collection}

\subsubsection{Growth Parameters}

Data were collected weekly on feed intake (FI), live body weight (LBW), from which, weight gain (WG) and feed conversion ratio (FCR) were calculated. At 49 days, 10 birds per treatment were randomly selected, fasted for 24 hours, weighed, slaughtered and eviscerated to evaluate carcass characteristics as proceeded by Kana et al. [13]. The length of the intestine was measured with the cut done from the start of the duodenal loop to the end of the cloacal. The density of the intestine was calculated by dividing the intestine weight by its length [13].

\subsubsection{Haemato-Biochemical Parameters and Histology of the Organs}

Blood was collected in heparinised and non-heparinised test tubes for the analysis of haematological and biochemical parameters respectively. Haematological parameters including white blood cell (WBC), red blood cell (RBC), haemoglobin $(\mathrm{Hb})$, haematocrit (HCT) and platelets (PLT) were analysed using Genius electronic haematocymeter (Model KT6180S/N 701106101557).

For biochemical analysis, blood samples were stored at room temperature and after 24 hours, the serum was collected and preserved at $-20^{\circ} \mathrm{C}$ for the evaluation of serum content in protein, albumin, globulin, triglyceride, total cholesterol, HDL and LDL-cholesterol, Aspartate aminotransferase (ASAT), Alamineaminotransferase (ALAT), urea and creatinine using Chronolab ${ }^{\circledR}$ commercial kits.

Liver and kidney samples were randomly sliced from each treatment and fixed by immersion in formol solution for 2 weeks. Tissues were dehydrated in graded levels of ethanol, xylene and embedded in paraffin. Sections of $5 \mu \mathrm{m}$ were stained with hematoxylin-eosine for histological observations (40× magnification).

\subsubsection{Microbial Count}

At the end of the trial, faeces samples were collected from the cloaca using an antiseptic scovel from 4 birds per treatment. The lactic acid bacteria, Escherichia coli and Salmonella counts were assessed in a specific culture media (MRS Agar for lactic acid bacteria, Mac Conkey Agar for E. coli and SS Agar for salmonella respectively) as proceeded by Ciza et al. [14].

\subsection{Statistical Analysis}

Data collected were submitted to a two-way analysis of variance using the Statistical Package for Social Science (SPSS 20.0). Significant differences between treatment means were separated using Duncan's Multiple Range test. Probability values less than 0.05 were considered significant. Student test was used to separate growth parameters of males and females.

\section{Results}

Table 2 summarized the effects of methenamine feeding regime on feed intake, live body weight, weight gain and feed conversion ratio. The results indicate no 
significant difference between the males and females. The inclusion of methenamine in water significantly increased feed intake (FI) irrespective of gender and feeding regime compared to the positive control $\left(\mathrm{T}^{+}\right)$. The administration of methenamine through non-acidify drinking water (TE) significantly increased body weight (BW) and weight gain (WG) while feed conversion ratio (FCR) significantly decreased compared to the control groups.

Table 3 summarizes carcass characteristics of broilers as affected by methenamine feeding regime. These results indicate no significant $(\mathrm{p}>0.05)$ effects among the treatment groups for carcass yields and relative weight of organs.

The development of digestive organs of broilers as affected by the inclusion of methenamine in feed, acidified and non acidified water is summarized in Table 4. Methenamine feeding regime had no significant ( $p>0.05)$ effects on the development of the digestive organs with respect to the negative and positive control treatments.

Table 2. Growth performance of broilers as affected by methenamine feeding regimes.

\begin{tabular}{|c|c|c|c|c|c|c|c|}
\hline & \multirow{2}{*}{ Sex } & \multicolumn{5}{|c|}{ Treatments } & \multirow{2}{*}{$p$} \\
\hline & & To & $\mathrm{T}^{+}$ & TA & $\mathrm{TE}$ & TEa & \\
\hline \multirow{4}{*}{$\begin{array}{c}\text { Feed } \\
\text { intake }(\mathrm{g})\end{array}$} & $\hat{0}$ & $3905.92 \pm 283.27^{\mathrm{a}}$ & $3533.20 \pm 352.65^{\mathrm{b}}$ & $3978.42 \pm 166.87^{\mathrm{a}}$ & $3960 \pm 273.13^{\mathrm{a}}$ & $3958.75 \pm 254.36^{\mathrm{a}}$ & 0.044 \\
\hline & q & $3786.80 \pm 389.40$ & $3511.50 \pm 378.14$ & $3845.83 \pm 236.38$ & $3796.42 \pm 215.34$ & $3563.80 \pm 279.06$ & 0.245 \\
\hline & ơo & $3846.36 \pm 330.56^{\mathrm{a}}$ & $3522.35 \pm 348.79^{\mathrm{b}}$ & $3912.13 \pm 207.00^{\mathrm{a}}$ & $3878.21 \pm 249.57^{\mathrm{a}}$ & $3761.28 \pm 327.64^{\mathrm{ab}}$ & 0.017 \\
\hline & $p$ & 0.829 & 0.994 & 0.546 & 0.531 & 0.098 & \\
\hline \multirow{4}{*}{$\begin{array}{c}\text { Body } \\
\text { weight }(g)\end{array}$} & $\hat{\sigma}$ & $2326.08 \pm 158.91^{c}$ & $2443.17 \pm 134.29^{b c}$ & $2513.67 \pm 132.96^{\mathrm{b}}$ & $2707.42 \pm 189.35^{\mathrm{a}}$ & $2544.92 \pm 104.02^{\mathrm{ab}}$ & 0.003 \\
\hline & q & $2131.33 \pm 158.42^{\mathrm{b}}$ & $2286.67 \pm 182.82^{\mathrm{b}}$ & $2421.75 \pm 179.86^{\mathrm{a}}$ & $2470.00 \pm 133.40^{\mathrm{a}}$ & $2376.17 \pm 291.85^{\mathrm{ab}}$ & 0.050 \\
\hline & ठoㅇ & $2228.71 \pm 182.29^{c}$ & $2364.92 \pm 173.41^{\mathrm{bc}}$ & $2467.71 \pm 158.25^{\mathrm{ab}}$ & $2588.71 \pm 199.40^{\mathrm{a}}$ & $2460.54 \pm 226.72^{\mathrm{ab}}$ & 0.000 \\
\hline & $p$ & 0.169 & 0.290 & 0.610 & 0.105 & 0.438 & \\
\hline \multirow{4}{*}{$\begin{array}{l}\text { Weight } \\
\text { gain (g) }\end{array}$} & $\hat{\sigma}$ & $1687.12 \pm 158.91^{c}$ & $1804.21 \pm 134.29^{b c}$ & $1874.71 \pm 132.96^{\mathrm{b}}$ & $2068.46 \pm 189.35^{\mathrm{a}}$ & $1905.96 \pm 104.02^{\mathrm{ab}}$ & 0.003 \\
\hline & q & $1547.57 \pm 158.42^{\mathrm{b}}$ & $1702.91 \pm 182.82^{\mathrm{ab}}$ & $1837.99 \pm 179.86^{\mathrm{a}}$ & $1886.24 \pm 133.40^{\mathrm{a}}$ & $1792.41 \pm 291.85^{\mathrm{ab}}$ & 0.050 \\
\hline & 구우 & $1617.35 \pm 167.92^{c}$ & $1753.56 \pm 161.83^{\mathrm{bc}}$ & $1856.35 \pm 152.01^{\mathrm{ab}}$ & $1977.35 \pm 182.87^{\mathrm{a}}$ & $1849.18 \pm 217.14^{\mathrm{ab}}$ & 0.000 \\
\hline & $p$ & 0.354 & 0.562 & 0.920 & 0.217 & 0.671 & \\
\hline \multirow{4}{*}{$\begin{array}{c}\text { Feed } \\
\text { concertion } \\
\text { ratio }\end{array}$} & $\hat{0}$ & $2.32 \pm 0.14^{\mathrm{a}}$ & $1.97 \pm 0.28^{\mathrm{b}}$ & $2.13 \pm 0.15^{\mathrm{ab}}$ & $1.93 \pm 0.26^{\mathrm{b}}$ & $2.08 \pm 0.19^{\mathrm{ab}}$ & 0.032 \\
\hline & q & $2.46 \pm 0.31^{\mathrm{a}}$ & $2.07 \pm 0.13^{\mathrm{b}}$ & $2.12 \pm 0.30^{\mathrm{b}}$ & $2.02 \pm 0.16^{\mathrm{b}}$ & $2.02 \pm 0.23^{\mathrm{b}}$ & 0.017 \\
\hline & $\widehat{\jmath} 0$ & $2.39 \pm 0.24^{\mathrm{a}}$ & $2.02 \pm 0.22^{\mathrm{b}}$ & $2.12 \pm 0.23^{b}$ & $1.98 \pm 0.21^{\mathrm{b}}$ & $2.05 \pm 0.21^{\mathrm{b}}$ & 0.000 \\
\hline & $p$ & 0.603 & 0.759 & 0.995 & 0.774 & 0.867 & \\
\hline
\end{tabular}

a,b,c Means with the same superscript on the same row are not significantly different $(\mathrm{p}>0.05)$. T0 = basal diet; T0 ${ }^{+}$: T0 + Antibiotic; $\mathrm{TA}$ : T0 $+\mathrm{Methenamine}$ in feed; TE: T0 + Methenamine in water; TEa: T0 + Methenamine in acidify water; $\overbrace{}^{\wedge}:$ male; $q$ : female. 
Table 3. Carcass yield and relative weight of organs of broilers as affected by methenamine feeding regimes.

\begin{tabular}{|c|c|c|c|c|c|c|c|}
\hline \multirow{2}{*}{$\begin{array}{c}\text { Characteristics } \\
\text { (\%bw) }\end{array}$} & \multirow{2}{*}{ Gender } & \multicolumn{5}{|c|}{ Treatments } & \multirow{2}{*}{$p$} \\
\hline & & T0 & $\mathrm{TO}^{+}$ & TA & $\mathrm{TE}$ & TEa & \\
\hline \multirow{4}{*}{$\begin{array}{l}\text { Carcass } \\
\text { yield }(\%)\end{array}$} & $\hat{\sigma}$ & $71.75 \pm 0.59$ & $72.51 \pm 1.27$ & $73.08 \pm 1.54$ & $73.27 \pm 1.54$ & $71.38 \pm 1.28$ & 0.132 \\
\hline & q & $72.56 \pm 1.32$ & $71.56 \pm 1.80$ & $73.02 \pm 0.84$ & $72.50 \pm 2.37$ & $72.41 \pm 2.81$ & 0.831 \\
\hline & $\widehat{o} q$ & $72.16 \pm 1.05$ & $72.03 \pm 1.55$ & $73.05 \pm 1.17$ & $72.89 \pm 1.93$ & $71.89 \pm 2.13$ & 0.392 \\
\hline & $p$ & 0.485 & 0.636 & 0.997 & 0.830 & 0.755 & \\
\hline \multirow{4}{*}{ Head } & $\hat{\sigma}$ & $2.23 \pm 0.22$ & $2.31 \pm 0.20$ & $2.10 \pm 0.30$ & $1.98 \pm 0.25$ & $2.19 \pm 0.22$ & 0.277 \\
\hline & ㅇ & $1.99 \pm 0.21$ & $1.97 \pm 0.12$ & $2.04 \pm 0.10$ & $1.98 \pm 0.21$ & $2.14 \pm 0.25$ & 0.570 \\
\hline & jo우 & $2.11 \pm 0.24$ & $2.14 \pm 0.24$ & $2.07 \pm 0.21$ & $1.98 \pm 0.22$ & $2.17 \pm 0.23$ & 0.425 \\
\hline & $p$ & 0.266 & 0.054 & 0.921 & 1.000 & 0.960 & \\
\hline \multirow{4}{*}{ Leg } & $\hat{\sigma}$ & $2.23 \pm 0.22^{\mathrm{B}}$ & $2.31 \pm 0.20^{\mathrm{B}}$ & $2.10 \pm 0.30^{\mathrm{B}}$ & $1.98 \pm 0.25^{\mathrm{B}}$ & $2.19 \pm 0.22^{\mathrm{B}}$ & 0.277 \\
\hline & q & $3.37 \pm 0.51^{\mathrm{A}}$ & $3.50 \pm 0.51^{\mathrm{A}}$ & $3.39 \pm 0.51^{\mathrm{A}}$ & $3.36 \pm 0.33^{\mathrm{A}}$ & $3.34 \pm 0.43^{\mathrm{A}}$ & 0.985 \\
\hline & $\lambda$ 우 & $2.80 \pm 0.71^{\mathrm{AB}}$ & $2.90 \pm 0.72^{\mathrm{AB}}$ & $2.75 \pm 0.79^{\mathrm{AB}}$ & $2.67 \pm 0.78^{\mathrm{AB}}$ & $2.76 \pm 0.69^{\mathrm{AB}}$ & 0.970 \\
\hline & $p$ & 0.022 & 0.019 & 0.018 & 0.007 & 0.015 & \\
\hline \multirow{4}{*}{ Liver } & $\hat{\sigma}$ & $1.70 \pm 0.19$ & $1.94 \pm 0.37$ & $1.79 \pm 0.23$ & $1.69 \pm 0.23$ & $1.83 \pm 0.28$ & 0.574 \\
\hline & q & $1.98 \pm 0.16$ & $2.14 \pm 0.29$ & $1.81 \pm 0.31$ & $1.95 \pm 0.29$ & $1.84 \pm 0.38$ & 0.430 \\
\hline & ๙o웅 & $1.84 \pm 0.22$ & $2.04 \pm 0.33$ & $1.80 \pm 0.25$ & $1.82 \pm 0.28$ & $1.84 \pm 0.32$ & 0.332 \\
\hline & $p$ & 0.114 & 0.621 & 0.990 & 0.334 & 0.999 & \\
\hline \multirow{4}{*}{ Heart } & o & $0.42 \pm 0.08$ & $0.50 \pm 0.09$ & $0.41 \pm 0.14$ & $0.49 \pm 0.09$ & $0.47 \pm 0.07$ & 0.453 \\
\hline & q & $0.47 \pm 0.08$ & $0.45 \pm 0.10$ & $0.45 \pm 0.08$ & $0.40 \pm 0.07$ & $0.52 \pm 0.04$ & 0.275 \\
\hline & đ우 & $0.45 \pm 0.08$ & $0.48 \pm 0.09$ & $0.43 \pm 0.11$ & $0.45 \pm 0.09$ & $0.49 \pm 0.06$ & 0.498 \\
\hline & $p$ & 0.542 & 0.706 & 0.828 & 0.302 & 0.533 & \\
\hline \multirow{4}{*}{ Pancréas } & $\widehat{\jmath}$ & $0.15 \pm 0.04$ & $0.17 \pm 0.03$ & $0.19 \pm 0.07$ & $0.18 \pm 0.05$ & $0.17 \pm 0.06$ & 0.719 \\
\hline & q & $0.15 \pm 0.05$ & $0.16 \pm 0.07$ & $0.17 \pm 0.06$ & $0.19 \pm 0.06$ & $0.19 \pm 0.09$ & 0.783 \\
\hline & $\delta^{10}+$ & $0.15 \pm 0.04$ & $0.16 \pm 0.05$ & $0.18 \pm 0.06$ & $0.19 \pm 0.05$ & $0.18 \pm 007$ & 0.550 \\
\hline & $p$ & 0.989 & 0.985 & 0.815 & 0.904 & 0.913 & \\
\hline \multirow{4}{*}{ Abdominal fat } & $\hat{\sigma}$ & $1.87 \pm 0.35$ & $1.76 \pm 0.40$ & $1.70 \pm 0.73$ & $1.83 \pm 0.15$ & $1.80 \pm 0.31$ & 0.973 \\
\hline & q & $1.72 \pm 0.47$ & $1.68 \pm 0.57$ & $1.92 \pm 0.38$ & $2.19 \pm 0.55$ & $1.64 \pm 0.44$ & 0.384 \\
\hline & $\delta^{\lambda}+$ & $1.79 \pm 0.40$ & $1.72 \pm 0.47$ & $1.81 \pm 0.56$ & $2.01 \pm 0.42$ & $1.72 \pm 0.37$ & 0.594 \\
\hline & $p$ & 0.838 & 0.972 & 0.833 & 0.405 & 0.794 & \\
\hline
\end{tabular}

A,B,C Means with the same superscript on the same colomn are not significantly different $(\mathrm{p}>0.05)$. T0 = basal diet; $\mathrm{T}^{+}: \mathrm{T} 0+$ Antibiotic; TA: T0 + Methenamine in feed; TE: T0 + Methenamine in water; TEa: T0 +Methenamine in acidify water; $\widehat{\partial}$ : male; ; : female. 
Table 4. Digestive organs of broilers as affected by methenamine feeding regimes.

\begin{tabular}{|c|c|c|c|c|c|c|c|}
\hline \multirow{2}{*}{ Organes } & \multirow{2}{*}{ Gender } & \multicolumn{5}{|c|}{ Treatments } & \multirow{2}{*}{$P$} \\
\hline & & T0 & $\mathrm{T}^{+}$ & TA & $\mathrm{TE}$ & $\mathrm{TEa}$ & \\
\hline \multirow{4}{*}{ Gizard (\%) } & $\hat{0}$ & $1.34 \pm 0.10$ & $1.25 \pm 0.11$ & $1.44 \pm 0.19$ & $1.49 \pm 0.19$ & $1.40 \pm 0.14$ & 0.157 \\
\hline & ㅇ & $1.39 \pm 0.15$ & $1.47 \pm 0.15$ & $1.35 \pm 0.16$ & $1.37 \pm 0.11$ & $1.42 \pm 0.35$ & 0.896 \\
\hline & $j 0$ & $1.36 \pm 0.12$ & $1.36 \pm 0.17$ & $1.39 \pm 0.17$ & $1.43 \pm 0.16$ & $1.41 \pm 0.25$ & 0.894 \\
\hline & $p$ & 0.778 & 0.101 & 0.729 & 0.538 & 0.994 & \\
\hline \multirow{4}{*}{$\begin{array}{l}\text { Intestinal weight } \\
(\mathrm{g})\end{array}$} & $\hat{0}$ & $69.75 \pm 10.54$ & $64.25 \pm 5.97^{\mathrm{A}}$ & $65.40 \pm 5.77$ & $72.00 \pm 6.04$ & $66.60 \pm 4.34$ & 0.389 \\
\hline & q & $56.60 \pm 4.56^{\mathrm{b}}$ & $54.50 \pm 2.18^{\mathrm{bB}}$ & $65.80 \pm 4.49^{\mathrm{a}}$ & $60.50 \pm 7.26^{\mathrm{ab}}$ & $60.20 \pm 4.55^{\mathrm{ab}}$ & 0.017 \\
\hline & $\delta$ ot & $63.18 \pm 10.33$ & $59.38 \pm 6.66^{\mathrm{AB}}$ & $65.60 \pm 4.88$ & $66.25 \pm 8.74$ & $63.40 \pm 5.38$ & 0.285 \\
\hline & $p$ & 0.114 & 0.050 & 0.992 & 0.097 & 0.156 & \\
\hline \multirow{4}{*}{$\begin{array}{l}\text { Intestinal lenght } \\
(\mathrm{cm})\end{array}$} & $\hat{0}$ & $204.50 \pm 10.62$ & $186.25 \pm 7.12$ & $206.18 \pm 11.43$ & $203.00 \pm 10.19$ & $191.00 \pm 23.47$ & 0.117 \\
\hline & 우 & $185.30 \pm 9.98$ & $182.88 \pm 13.25$ & $188.22 \pm 16.27$ & $184.88 \pm 18.18$ & $192.38 \pm 4.05$ & 0.818 \\
\hline & jo & $194.90 \pm 14.03$ & $184.56 \pm 10.18$ & $197.20 \pm 16.29$ & $193.94 \pm 16.86$ & $191.69 \pm 15.90$ & 0.387 \\
\hline & $p$ & 0.077 & 0.878 & 0.207 & 0.226 & 0.991 & \\
\hline \multirow{4}{*}{$\begin{array}{l}\text { Intestinal density } \\
\qquad(\mathrm{g} / \mathrm{cm})\end{array}$} & $\widehat{o}$ & $0.34 \pm 0.05$ & $0.34 \pm 0.03$ & $0.32 \pm 0.04$ & $0.34 \pm 0.02$ & $0.34 \pm 0.07$ & 0.872 \\
\hline & 우 & $0.31 \pm 0.03$ & $0.30 \pm 0.03$ & $0.35 \pm 0.04$ & $0.33 \pm 0.06$ & $0.32 \pm 0.03$ & 0.301 \\
\hline & jot & $0.32 \pm 004$ & $0.32 \pm 0.04$ & $0.33 \pm 0.04$ & $0.34 \pm 0.04$ & $0.33 \pm 0.05$ & 0.925 \\
\hline & $p$ & 0.440 & 0.112 & 0.402 & 0.947 & 0.902 & \\
\hline
\end{tabular}

${ }^{a, b}$ Means with the same superscript on the same row are not significantly different $(p>0.05) ;{ }^{A, B}$ Means with the same superscript on the same colomn are not significantly different $(\mathrm{p}>0.05)$. T0 = basal diet; $\mathrm{T} 0^{+}: \mathrm{T} 0+$ Antibiotic; TA: T0 + Methenamine in feed; TE: T0 + Methenamine in water; TEa: T0 + Methe-

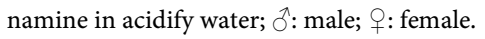

The microbes isolated from the faecal sample of growing broilers fed on methenamine are shown in Table 5. Feeding broilers with methenamine through feed, acidified and non acidified drinking water resulted to a significant increased in the lactic acid bacteria count. Treatments groups were comparables for E. coli count, while Salmonella was absent in all the groups.

Figure 1 illustrates the variation of coccidia count with respect to methenamine feeding regime. Coccidia count decreases in all treatment groups during the first two weeks except in broilers fed on methenamine through acidified water. However, they were absent in birds fed on the positive control diets and those fed on methenamine in non acidified water in the third week of trial. Coccidia were totally absent in all groups at the last week of the trial.

Except for the WBC and PLT concentration that significantly $(\mathrm{p}<0.05)$ decrease in males fed on methenamine through feed compared to the other treatments. Haematological parameters of broilers were not significantly $(p>0.05)$ affected whatever the treatments (Table 6). 
Table 5. Gut microbial load of broilers fed on methenamine feeding regimes.

\begin{tabular}{|c|c|c|c|c|c|c|}
\hline \multirow{2}{*}{$\begin{array}{l}\text { Bacteria load } \\
\text { (Log10CFU) }\end{array}$} & \multicolumn{5}{|c|}{ Treatments } & \multirow{2}{*}{$p$} \\
\hline & T0 & $\mathrm{TO}^{+}$ & TA & TE & TEa & \\
\hline Lactobacillus & $9.52 \pm 0.09^{\mathrm{b}}$ & $9.60 \pm 0.09^{\mathrm{ab}}$ & $9.72 \pm 0.04^{\mathrm{a}}$ & $9.63 \pm 0.16^{\mathrm{ab}}$ & $9.72 \pm 0.06^{\mathrm{a}}$ & 0.045 \\
\hline Coliforms & $9.35 \pm 0.23^{\mathrm{a}}$ & $9.26 \pm 0.04^{\mathrm{ab}}$ & $9.39 \pm 0.15^{\mathrm{a}}$ & $9.46 \pm 0.18^{\mathrm{a}}$ & $8.89 \pm 0.45^{\mathrm{b}}$ & 0.045 \\
\hline Salmonella & 0.00 & 0.00 & 0.00 & 0.00 & 0.00 & \\
\hline
\end{tabular}

${ }^{a, b}$ Means with the same superscript on the same row are not significantly different $(\mathrm{p}>0.05)$; $\mathrm{T} 0=$ basal diet; $\mathrm{T} 0^{+}: \mathrm{T} 0+\mathrm{Antibiotic}$; $\mathrm{TA}$ : T0 + Methenamine in feed; TE: T0 + Methenamine in water; TEa: T0 + Methenamine in acidify water.

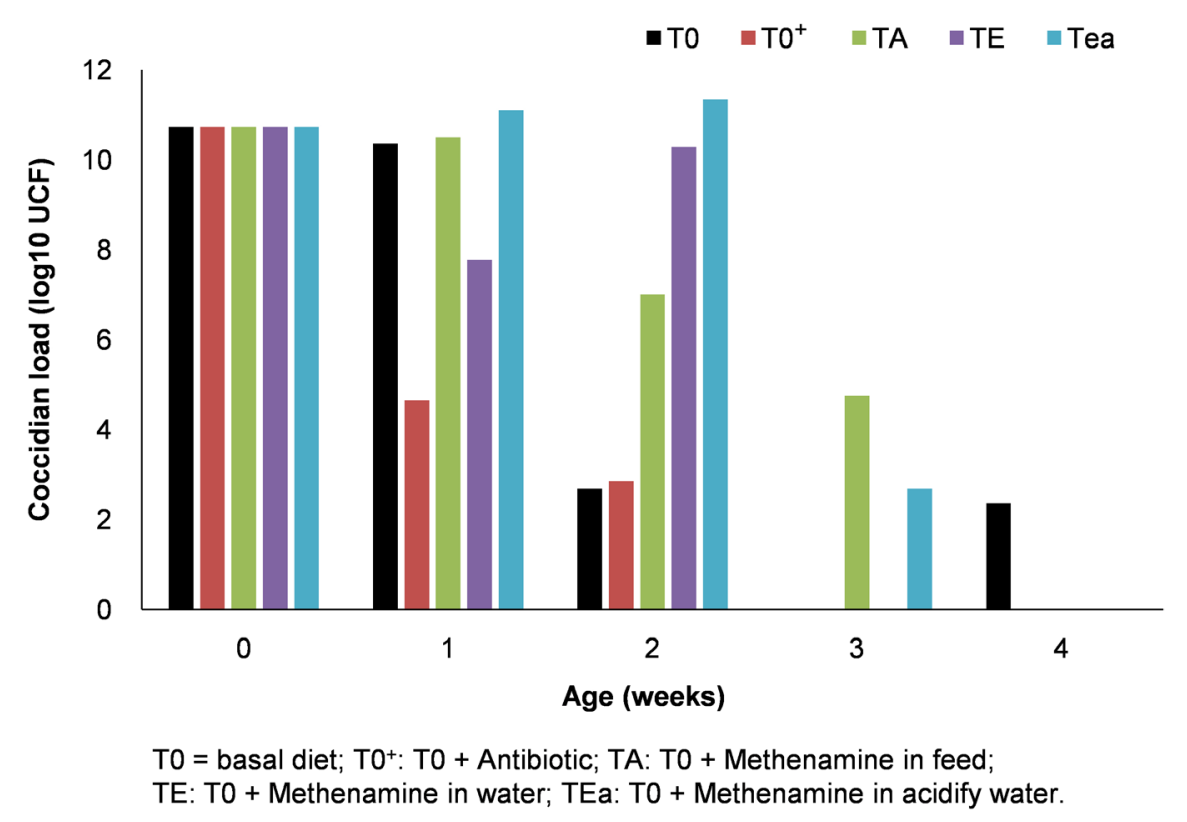

Figure 1. Coccidian load as affected by methenamine feeding regimes.

The biochemical parameters of broilers as affected by methenamine feeding regime are summarized in Table 7 . Serum content in ASAT and ALAT significantly decreased irrespective of the feeding regime and gender compared to the negative control diet (T0). Serum content in creatinine and urea significantly ( $p$ $<0.05)$ decreased in male compared to the negative and positive control groups. Total protein and globulin decreased significantly while albumin/globulin ratio increased in broilers fed on methenamine through drinking water irrespective of gender compared to the control groups. Serum concentration of total and LDL-cholesterol significantly decreased while HDL-cholesterol increased with methenamine in non acidified drinking water (Table 8).

Histological structures of liver and kidney are presented in Figure 2 and Figure 3. Methenamine feeding regime induced steatose in liver of broiler chickens. the kidneys show that animals treated through feed and acidified water have a disorganized structure in which the structures of the glomerular were almost indistinguishable and also steatosis characterized by the dilation of the inter glomerular spaces. 
Table 6. Haematological parameters of broilers as affected by methenamine feeding regimes.

\begin{tabular}{|c|c|c|c|c|c|c|c|}
\hline \multirow{2}{*}{ Parameters } & \multirow{2}{*}{ Gender } & \multicolumn{5}{|c|}{ Treatments } & \multirow{2}{*}{$\mathrm{P}$} \\
\hline & & T0 & T0+ & TA & TE & TEa & \\
\hline \multirow{4}{*}{$\mathrm{WBC}\left(10^{3} / \mu \mathrm{L}\right)$} & $\hat{0}$ & $93.70 \pm 3.60^{\mathrm{a}}$ & $90.40 \pm 3.17^{\mathrm{ab}}$ & $79.60 \pm 8.20^{c}$ & $82.60 \pm 6.74^{\mathrm{bc}}$ & $88.03 \pm 0.74^{\mathrm{abc}}$ & 0.045 \\
\hline & 우 & $79.97 \pm 1.89$ & $84.03 \pm 0.76$ & $80.90 \pm 6.79$ & $83.93 \pm 1.89$ & $90.07 \pm 8.84$ & 0.211 \\
\hline & $\widehat{\partial}$ 우 & $86.83 \pm 7.95$ & $87.22 \pm 4.05$ & $80.25 \pm 6.77$ & $83.27 \pm 4.48$ & $89.05 \pm 5.72$ & 0.112 \\
\hline & $p$ & 0.069 & 0.125 & 0.975 & 0.942 & 0.917 & \\
\hline \multirow{4}{*}{$\operatorname{RBC}\left(10^{6} / \mu \mathrm{L}\right)$} & $\hat{0}$ & $2.91 \pm 0.82$ & $3.09 \pm 0.21$ & $2.46 \pm 0.49$ & $2.59 \pm 0.31$ & $2.60 \pm 0.27$ & 0.492 \\
\hline & q & $2.53 \pm 0.19$ & $2.89 \pm 0.15$ & $2.63 \pm 0.39$ & $2.80 \pm 0.31$ & $2.53 \pm 0.38$ & 0.494 \\
\hline & $\delta P$ & $2.72 \pm 057$ & $2.99 \pm 0.20$ & $2.54 \pm 0.41$ & $2.70 \pm 0.30$ & $2.56 \pm 030$ & 0.273 \\
\hline & $p$ & 0.728 & 0.463 & 0.891 & 0.713 & 0.960 & \\
\hline \multirow{4}{*}{ HGB (g/dL) } & $\sigma^{\pi}$ & $16.40 \pm 1.60$ & $14.07 \pm 1.03$ & $12.47 \pm 2.00$ & $12.80 \pm 1.83$ & $13.13 \pm 0.70$ & 0.058 \\
\hline & 우 & $12.40 \pm 0.20$ & $13.73 \pm 1.01$ & $12.80 \pm 1.91$ & $13.53 \pm 1.53$ & $12.13 \pm 1.15$ & 0.514 \\
\hline & o우 & $14.40 \pm 2.42$ & $13.90 \pm 0.93$ & $12.63 \pm 1.76$ & $13.17 \pm 1.56$ & $12.63 \pm 1.02$ & 0.265 \\
\hline & $p$ & 0.092 & 0.91 & 0.976 & 0.860 & 0.493 & \\
\hline \multirow{4}{*}{ HCT (\%) } & $\pi$ & $44.80 \pm 5.80$ & $39.40 \pm 3.20$ & $33.67 \pm 7.37$ & $36.93 \pm 5.06$ & $35.27 \pm 2.73$ & 0.147 \\
\hline & q & $35.73 \pm 2.90$ & $37.73 \pm 1.40$ & $32.10 \pm 1.70$ & $36.47 \pm 4.32$ & $33.80 \pm 3.86$ & 0.251 \\
\hline & o우 & $40.27 \pm 6.44$ & $38.57 \pm 2.39$ & $32.88 \pm 4.86$ & $36.70 \pm 4.22$ & $34.53 \pm 3.09$ & 0.053 \\
\hline & $p$ & 0.204 & 0.711 & 0.932 & 0.992 & 0.857 & \\
\hline \multirow{4}{*}{$\mathrm{CMH}(\mathrm{pg})$} & $\pi$ & $48.57 \pm 1.05$ & $45.40 \pm 0.26$ & $50.93 \pm 2.90$ & $49.23 \pm 1.88$ & $50.70 \pm 3.87$ & 0.097 \\
\hline & 우 & $48.97 \pm 3.53$ & $47.37 \pm 1.01$ & $48.77 \pm 3.00$ & $48.30 \pm 0.26$ & $48.27 \pm 3.05$ & 0.944 \\
\hline & $\delta$ oㅇ & $48.77 \pm 2.34$ & $46.38 \pm 1.26$ & $49.85 \pm 2.90$ & $48.77 \pm 1.30$ & $49.48 \pm 3.39$ & 0.140 \\
\hline & $p$ & 0.980 & 0.130 & 0.674 & 0.698 & 0.696 & \\
\hline \multirow{4}{*}{$\mathrm{MCHC}(\mathrm{g} / \mathrm{dL})$} & $\hat{\sigma}$ & $35.63 \pm 2.20$ & $35.67 \pm 0.70$ & $37.37 \pm 2.56$ & $34.60 \pm 0.60$ & $37.30 \pm 2.59$ & 0.397 \\
\hline & q & $34.83 \pm 3.23$ & $36.33 \pm 2.03$ & $35.90 \pm 1.41$ & $37.07 \pm 0.50$ & $35.90 \pm 0.92$ & 0.698 \\
\hline & oo & $35.23 \pm 2.51$ & $36.00 \pm 1.41$ & $36.63 \pm 2.01$ & $35.83 \pm 1.44$ & $36.60 \pm 1.90$ & 0.690 \\
\hline & $p$ & 0.933 & 0.857 & 0.689 & 0.073 & 0.682 & \\
\hline \multirow{4}{*}{ PLT (fL) } & $\sigma^{\lambda}$ & $62.50 \pm 10.50^{\mathrm{bc}}$ & $94.50 \pm 2.50^{\mathrm{a}}$ & $70.00 \pm 18.00^{\mathrm{b}}$ & $44.00 \pm 6.56^{c}$ & $47.67 \pm 4.04^{c}$ & 0.001 \\
\hline & 우 & $50.50 \pm 10.50^{\mathrm{b}}$ & $54.00 \pm 7.00^{\mathrm{b}}$ & $56.00 \pm 9.64^{\mathrm{b}}$ & $104.50 \pm 12.50^{\mathrm{a}}$ & $53.00 \pm 10.15^{\mathrm{b}}$ & 0.000 \\
\hline & o우 & $56.50 \pm 11.46$ & $74.25 \pm 22.68$ & $63.00 \pm 15.02$ & $74.25 \pm 34.32$ & $50.33 \pm 7.50$ & 0.201 \\
\hline & $P$ & 0.446 & 0.053 & 0.533 & 0.059 & 0.701 & \\
\hline \multirow{4}{*}{ PCV (\%) } & $\hat{0}$ & $0.08 \pm 0.02$ & $0.10 \pm 0.02$ & $0.08 \pm 0.02$ & $0.07 \pm 0.01$ & $0.08 \pm 0.01$ & 0.282 \\
\hline & 우 & $0.12 \pm 0.06$ & $0.83 \pm 0.01$ & $0.83 \pm 0.15$ & $0.12 \pm 0.03$ & $0.80 \pm 0.01$ & 0.279 \\
\hline & o우 & $0.10 \pm 0.04$ & $0.09 \pm 0.01$ & $0.08 \pm 0.01$ & $0.10 \pm 0.03 b$ & $0.08 \pm 0.01$ & 0.563 \\
\hline & $p$ & 0.664 & 0.382 & 1.000 & 0.164 & 1.000 & \\
\hline
\end{tabular}

a,b,c Means with the same superscript on the same row are not significantly different $(\mathrm{p}>0.05)$. T0 = basal diet; $\mathrm{T} 0^{+}: \mathrm{T} 0+$ Antibiotic; $\mathrm{TA}: \mathrm{T} 0+\mathrm{Methenamine}$ in feed; TE: T0 + Methenamine in water; TEa: T0 +Methenamine in acidify water; ${ }^{2}$ : male; ; : female; $\mathrm{p}=$ Probability; WBC = White Blood Cell; RBC = Red blood cell; $\mathrm{Hgb}=$ Haemoglobin; $\mathrm{MCV}=$ Mean corpuscular volume; $\mathrm{MCH}=$ Mean corpuscular haemoglobin; $\mathrm{MCHC}=$ Mean corpuscular haemoglobin concentration; PCV = Packed cell volume; HCT = Hematocrit; PLT: Platelets. 
Table 7. Biochemical parameters of broilers as affected by methenamine feeding regimes.

\begin{tabular}{|c|c|c|c|c|c|c|c|}
\hline \multirow{2}{*}{ Parameters } & \multirow{2}{*}{ Gender } & \multicolumn{5}{|c|}{ Treatments } & \multirow{2}{*}{$\mathrm{p}$} \\
\hline & & To & $\mathrm{T}^{+}$ & TA & $\mathrm{TE}$ & $\mathrm{TEa}$ & \\
\hline \multirow{4}{*}{ ASAT (IU/L) } & $\hat{\sigma}$ & $246.75 \pm 170.98$ & $159.08 \pm 65.30$ & $194.91 \pm 64.67$ & $161.00 \pm 50.33$ & $180.95 \pm 55.51$ & 0.584 \\
\hline & 우 & $310.63 \pm 168.59^{a}$ & $149.63 \pm 51.76^{b}$ & $132.42 \pm 15.09^{\mathrm{b}}$ & $149.98 \pm 62.06^{b}$ & $102.81 \pm 38.47^{\mathrm{b}}$ & 0.013 \\
\hline & o우 & $282.24 \pm 162.20^{\mathrm{a}}$ & $154.35 \pm 55.78^{\mathrm{b}}$ & $167.13 \pm 57.10^{\mathrm{b}}$ & $155.49 \pm 53.59^{\mathrm{b}}$ & $141.88 \pm 61.02^{\mathrm{b}}$ & 0.007 \\
\hline & $p$ & 0.849 & 0.967 & 0.256 & 0.951 & 0.111 & \\
\hline \multirow{4}{*}{ ALAT (IU/L) } & $\hat{0}$ & $64.46 \pm 26.64^{\mathrm{a}}$ & $32.81 \pm 18.54^{\mathrm{bc}}$ & $59.15 \pm 29.44^{\mathrm{ab}}$ & $43.53 \pm 20.56^{\mathrm{abc}}$ & $24.28 \pm 6.53^{c}$ & 0.050 \\
\hline & 우 & $74.16 \pm 35.99^{\mathrm{a}}$ & $45.28 \pm 28.00^{\mathrm{ab}}$ & $23.63 \pm 8.77^{\mathrm{b}}$ & $35.66 \pm 19.53^{\mathrm{b}}$ & $42.00 \pm 20.95^{\mathrm{ab}}$ & 0.046 \\
\hline & $\delta$ of & $69.85 \pm 30.66^{\mathrm{a}}$ & $39.05 \pm 23.33^{\mathrm{b}}$ & $41.39 \pm 27.75^{\mathrm{b}}$ & $39.59 \pm 19.36^{\mathrm{b}}$ & $33.14 \pm 17.36^{\mathrm{b}}$ & 0.018 \\
\hline & $p$ & 0.900 & 0.709 & 0.111 & 0.821 & 0.265 & \\
\hline \multirow{4}{*}{$\begin{array}{l}\text { Creatinin } \\
(\mathrm{mg} / \mathrm{dL})\end{array}$} & $\hat{0}$ & $0.02 \pm 0.01^{\mathrm{a}}$ & $0.03 \pm 0.03^{b}$ & $0.01 \pm 0.00^{\mathrm{b}}$ & $0.02 \pm 0.00^{\mathrm{b}}$ & $0.02 \pm 0.01^{b}$ & 0.020 \\
\hline & 우 & $0.02 \pm 0.01^{\mathrm{b}}$ & $0.01 \pm 0.00^{\mathrm{b}}$ & $0.01 \pm 0.01^{\mathrm{b}}$ & $0.02 \pm 0.00^{\mathrm{b}}$ & $0.03 \pm 0.01^{\mathrm{a}}$ & 0.003 \\
\hline & oo & $0.02 \pm 0.01$ & $0.02 \pm 0.01$ & $0.01 \pm 0.00$ & $0.02 \pm 0.00$ & $0.02 \pm 0.01$ & 0.080 \\
\hline & $p$ & 0.874 & 0.092 & 1.000 & 0.357 & 0.105 & \\
\hline \multirow{4}{*}{ Urea $(\mathrm{mg} / \mathrm{dL})$} & $\hat{0}$ & $6.38 \pm 0.44^{\mathrm{bc}}$ & $7.76 \pm 0.28^{\mathrm{a}}$ & $7.67 \pm 0.91^{\mathrm{a}}$ & $7.23 \pm 1.14^{\mathrm{ab}}$ & $6.08 \pm 0.63^{c}$ & 0.008 \\
\hline & q & $5.84 \pm 0.96$ & $6.37 \pm 0.94$ & $6.68 \pm 0.81$ & $5.58 \pm 1.14$ & $6.23 \pm 0.56$ & 0.379 \\
\hline & ơ & $6.08 \pm 0.78^{b}$ & $7.06 \pm 0.98^{\mathrm{ab}}$ & $7.17 \pm 0.97^{\mathrm{a}}$ & $6.41 \pm 1.38^{\mathrm{ab}}$ & $6.15 \pm 0.57^{\mathrm{b}}$ & 0.050 \\
\hline & $p$ & 0.594 & 0.064 & 0.259 & 0.154 & 0.929 & \\
\hline \multirow{4}{*}{$\begin{array}{l}\text { Total proteins } \\
\qquad(\mathrm{g} / \mathrm{dL})\end{array}$} & $\hat{0}$ & $3.68 \pm 0.46^{\mathrm{a}}$ & $3.57 \pm 0.28^{\mathrm{ab}}$ & $3.90 \pm 0.43^{\mathrm{a}}$ & $3.50 \pm 0.28^{\mathrm{ab}}$ & $3.12 \pm 0.37^{b}$ & 0.045 \\
\hline & 우 & $3.69 \pm 0.32$ & $3.48 \pm 0.30$ & $3.27 \pm 0.37$ & $3.43 \pm 0.30$ & $3.26 \pm 0.13$ & 0.178 \\
\hline & oo & $3.68 \pm 0.36^{\mathrm{a}}$ & $3.53 \pm 0.28^{\mathrm{a}}$ & $3.59 \pm 0.50^{\mathrm{a}}$ & $3.47 \pm 0.28^{\mathrm{ab}}$ & $3.19 \pm 0.27^{b}$ & 0.039 \\
\hline & $p$ & 0.999 & 0.894 & 0.124 & 0.927 & 0.745 & \\
\hline \multirow{4}{*}{$\begin{array}{l}\text { Albumin } \\
(\mathrm{g} / \mathrm{dL})\end{array}$} & $\hat{0}$ & $1.65 \pm 0.32$ & $1.53 \pm 0.19$ & $1.68 \pm 0.13$ & $1.88 \pm 0.20$ & $1.89 \pm 0.25$ & 0.093 \\
\hline & 우 & $1.71 \pm 0.23$ & $1.67 \pm 0.18$ & $1.78 \pm 0.24$ & $1.75 \pm 0.11$ & $1.86 \pm 0.08$ & 0.519 \\
\hline & $\delta p$ & $1.68 \pm 0.26^{\mathrm{b}}$ & $1.60 \pm 0.19^{\mathrm{b}}$ & $1.73 \pm 0.19^{\mathrm{ab}}$ & $1.82 \pm 0.17^{\mathrm{a}}$ & $1.87 \pm 0.18^{\mathrm{a}}$ & 0.031 \\
\hline & $p$ & 0.948 & 0.541 & 0.671 & 0.470 & 0.969 & \\
\hline \multirow{4}{*}{$\begin{array}{l}\text { Globulin } \\
(\mathrm{g} / \mathrm{dL})\end{array}$} & $\hat{0}$ & $2.03 \pm 0,19^{\mathrm{ab}}$ & $2.04 \pm 0.32^{\mathrm{ab}}$ & $2.23 \pm 0.51^{\mathrm{a}}$ & $1.62 \pm 0.18^{\mathrm{bc}}$ & $1.24 \pm 0.26^{c}$ & 0.001 \\
\hline & q & $1.98 \pm 0,16^{\mathrm{a}}$ & $1.82 \pm 0.24^{\mathrm{ab}}$ & $1.49 \pm 0.19^{\mathrm{cd}}$ & $1.68 \pm 0.26^{b c}$ & $1.40 \pm 0.12^{\mathrm{d}}$ & 0.001 \\
\hline & o우 & $2.00 \pm 0.17^{\mathrm{a}}$ & $1.93 \pm 0.29^{\mathrm{ab}}$ & $1.86 \pm 0.53^{\mathrm{ab}}$ & $1.65 \pm 0.21^{\mathrm{b}}$ & $1.32 \pm 0.21^{\mathrm{c}}$ & 0.000 \\
\hline & $P$ & 0.923 & 0.503 & 0.070 & 0.907 & 0.458 & \\
\hline \multirow{4}{*}{ Ratio A/G } & $\widehat{0}$ & $0.81 \pm 0.14^{c}$ & $0.77 \pm 0.17^{c}$ & $0.80 \pm 0.29^{c}$ & $1.17 \pm 0.17^{\mathrm{b}}$ & $1.57 \pm 0.36^{\mathrm{a}}$ & 0.000 \\
\hline & q & $0.87 \pm 0.11^{\mathrm{b}}$ & $0.93 \pm 0.15^{\mathrm{b}}$ & $1.20 \pm 0.15^{\mathrm{ab}}$ & $1.06 \pm 0.18^{\mathrm{bc}}$ & $1.33 \pm 0.14^{\mathrm{a}}$ & 0.000 \\
\hline & ơ & $0.84 \pm 0.12^{c}$ & $0.85 \pm 0.17^{c}$ & $1.00 \pm 0.30^{b c}$ & $1.12 \pm 0.17^{\mathrm{b}}$ & $1.45 \pm 0.29^{\mathrm{a}}$ & 0.000 \\
\hline & $p$ & 0.823 & 0.350 & 0.091 & 0.627 & 0.426 & \\
\hline
\end{tabular}

a,b,c Means with the same superscript on the same row are not significantly different $(\mathrm{p}>0.05)$. T0 = basal diet; T0 ${ }^{+}$T0 + Antibiotic; TA: T0 + Methenamine in feed; TE: T0 + Methenamine in water; TEa: T0 +Methenamine in acidify water; $\mathrm{p}=$ Probability; $\delta$ : male; $ᄋ$ : female. 
Table 8. Atheromatous risks markers of broilers as affected by methenamine feeding regimes.

\begin{tabular}{|c|c|c|c|c|c|c|c|}
\hline \multirow{2}{*}{ Paramètres } & \multirow{2}{*}{ Gender } & \multicolumn{5}{|c|}{ Treatments } & \multirow{2}{*}{$p$} \\
\hline & & T0 & $\mathrm{T}^{+}$ & TA & TE & TEa & \\
\hline \multirow{4}{*}{$\begin{array}{l}\text { Triglycérides } \\
(\mathrm{mg} / \mathrm{dL})\end{array}$} & $\widehat{0}$ & $30.47 \pm 9.10$ & $28.79 \pm 6.11$ & $28.48 \pm 6.61$ & $23.78 \pm 9.32$ & $34.09 \pm 20.65$ & 0.729 \\
\hline & 우 & $15.41 \pm 3.96^{\mathrm{b}}$ & $22.17 \pm 6.35^{\mathrm{b}}$ & $40.14 \pm 16.08^{\mathrm{a}}$ & $16.73 \pm 6.80^{\mathrm{b}}$ & $26.44 \pm 8.02^{\mathrm{b}}$ & 0.005 \\
\hline & jo우 & $22.10 \pm 10.09$ & $25.48 \pm 6.84$ & $34.31 \pm 13.12$ & $20.64 \pm 8.64$ & $30.27 \pm 15.07$ & 0.057 \\
\hline & $p$ & 0.063 & 0.306 & 0.372 & 0.483 & 0.784 & \\
\hline \multirow{4}{*}{$\begin{array}{l}\text { Total cholestérol } \\
(\mathrm{mg} / \mathrm{dL})\end{array}$} & $\widehat{0}$ & $112.41 \pm 15.15^{\mathrm{ab}}$ & $121.52 \pm 14.68^{\mathrm{a}}$ & $103.77 \pm 8.94^{\mathrm{ab}}$ & $95.89 \pm 11.23^{\mathrm{b}}$ & $100.00 \pm 14.21^{\mathrm{b}}$ & 0.040 \\
\hline & q & $105.57 \pm 22.63$ & $111.59 \pm 16.72$ & $105.14 \pm 17.17$ & $96.32 \pm 11.62$ & $101.24 \pm 8.89$ & 0.677 \\
\hline & ¡우 & $108.61 \pm 18.84^{\mathrm{ab}}$ & $116.56 \pm 15.73^{\mathrm{a}}$ & $104.45 \pm 12.93^{\mathrm{ab}}$ & $96.11 \pm 10.77^{b}$ & $100.55 \pm 11.45^{\mathrm{b}}$ & 0.029 \\
\hline & $p$ & 0.871 & 0.616 & 0.987 & 0.998 & 0.988 & \\
\hline \multirow{4}{*}{$\begin{array}{l}\text { HDL-cholestérol } \\
\text { (mg/dL) }\end{array}$} & $\widehat{\sigma}$ & $50.43 \pm 7.33^{c}$ & $89.52 \pm 14.60^{\mathrm{a}}$ & $68.46 \pm 13.73^{\mathrm{b}}$ & $78.24 \pm 11.82^{\mathrm{ab}}$ & $76.35 \pm 5.14^{\mathrm{ab}}$ & 0.001 \\
\hline & 우 & $64.67 \pm 10.06^{\mathrm{b}}$ & $78.32 \pm 9.10^{\mathrm{a}}$ & $84.79 \pm 9.85^{\mathrm{a}}$ & $78.34 \pm 7.00^{\mathrm{a}}$ & $76.05 \pm 5.58^{\mathrm{ab}}$ & 0.023 \\
\hline & $\delta$ or & $58.34 \pm 11.27^{\mathrm{b}}$ & $83.92 \pm 12.90^{\mathrm{a}}$ & $76.63 \pm 14.17^{\mathrm{a}}$ & $78.29 \pm 9.16^{\mathrm{a}}$ & $76.21 \pm 4.99^{\mathrm{a}}$ & 0.000 \\
\hline & $p$ & 0.153 & 0.391 & 0.177 & 1.000 & 0.996 & \\
\hline \multirow{4}{*}{$\begin{array}{l}\text { LDL-cholestérol } \\
(\mathrm{mg} / \mathrm{dL})\end{array}$} & $\widehat{\sigma}$ & $68.07 \pm 17.21^{\mathrm{a}}$ & $37.76 \pm 21.56^{\mathrm{b}}$ & $41.00 \pm 17.89^{\mathrm{b}}$ & $22.40 \pm 9.98^{\mathrm{b}}$ & $29.11 \pm 8.29^{\mathrm{b}}$ & 0.005 \\
\hline & q & $43.98 \pm 29.47$ & $37.70 \pm 12.16$ & $31.93 \pm 19.14$ & $20.66 \pm 13.86$ & $24.39 \pm 18.00$ & 0.344 \\
\hline & $\hat{o}$ 우 & $54.69 \pm 26.58^{\mathrm{a}}$ & $37.73 \pm 16.50^{\mathrm{b}}$ & $36.47 \pm 18.11^{\mathrm{b}}$ & $21.53 \pm 11.42^{\mathrm{b}}$ & $26.75 \pm 13.44^{\mathrm{b}}$ & 0.003 \\
\hline & $p$ & 0.403 & 1.000 & 0.740 & 0.973 & 0.863 & \\
\hline
\end{tabular}

${ }^{\mathrm{a}, \mathrm{b}, \mathrm{c}}$ Means with the same superscript on the same row are not significantly different $(\mathrm{p}>0.05)$. T0 = basal diet; T0 ${ }^{+}$T0 $+\mathrm{Antibiotic}$; $\mathrm{TA}$ : T0 $+\mathrm{Methenamine}$ in feed; TE: T0 + Methenamine in water; TEa: T0 +Methenamine in acidify water; $\mathrm{p}=$ Probability; $\delta^{\jmath}:$ male; $\circ:$ female.
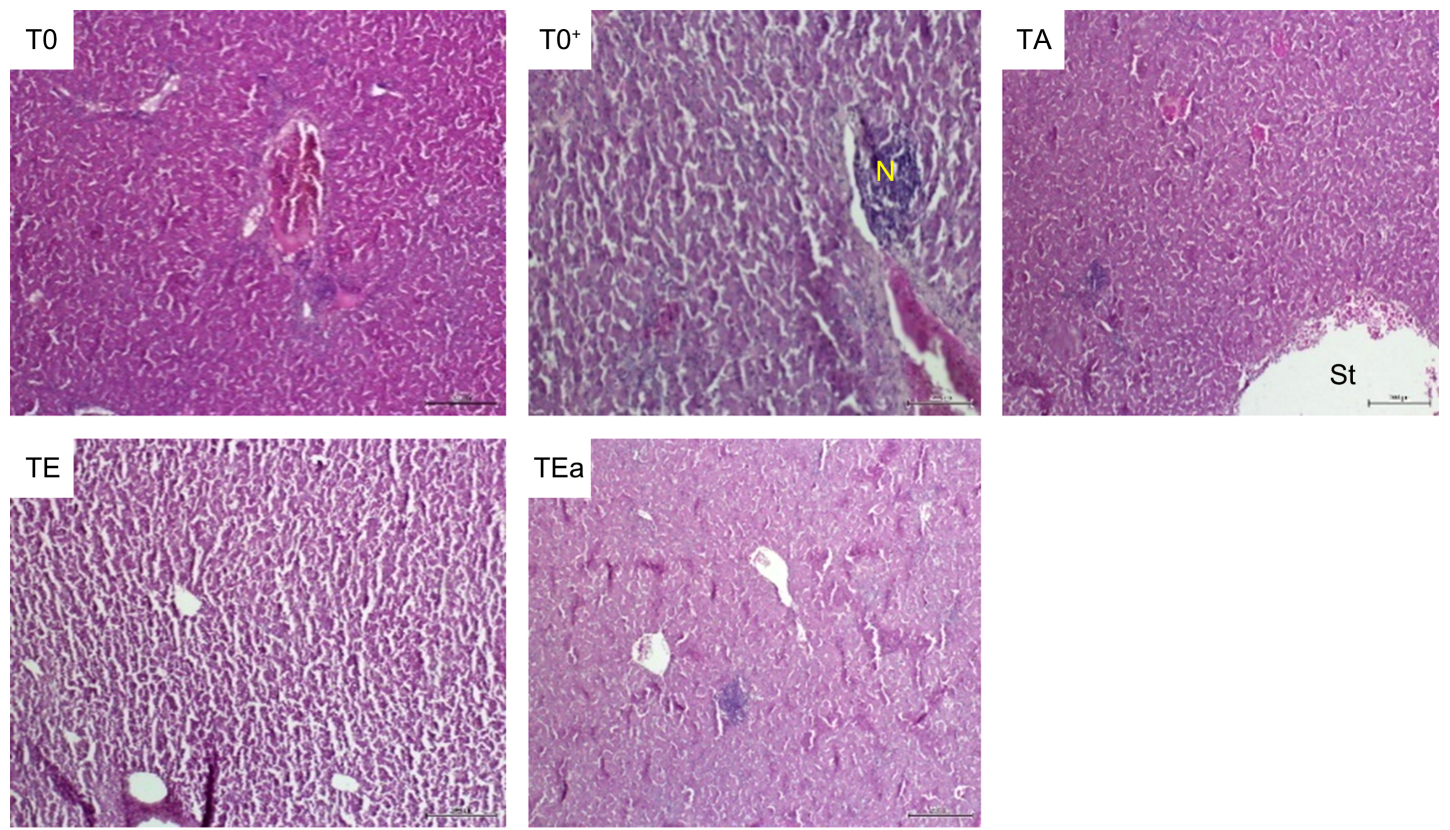

T0 = basal diet; $\mathrm{TO}^{+}: \mathrm{TO}+$ Antibiotic; TA: T0 + Methenamine in feed; TE: T0 + Methenamine in water;

TEa: T0 + Methenamine in acidify water; $p=$ Probabilit; N: Necrose; St: Stéatose; Grossissement: 400X.

Figure 2. Histological structure of the liver of broiler chickens as affected by methenamine feeding regimes. 

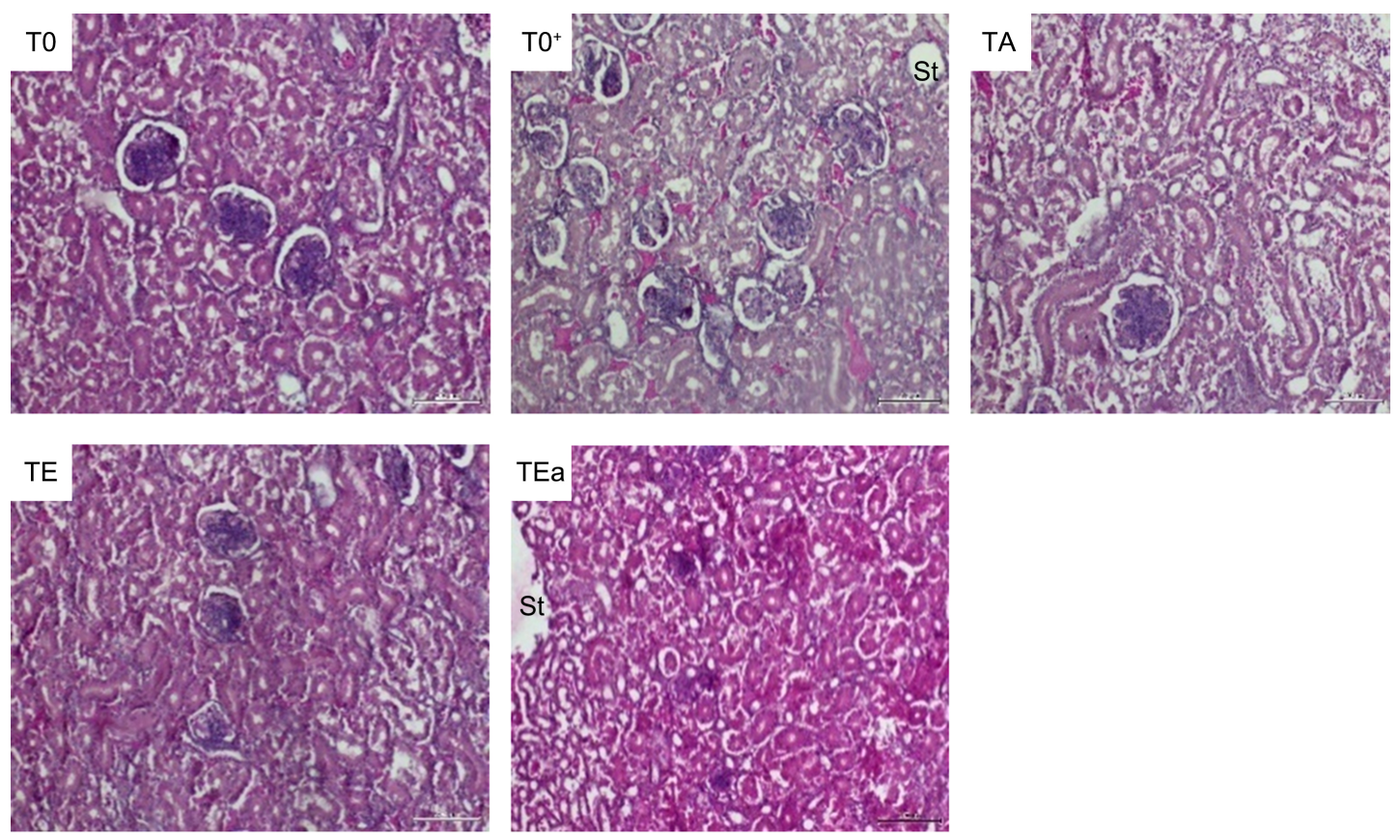

T0 = basal diet; TO+: T0 + Antibiotic; TA: T0 + Methenamine in feed; TE: T0 + Methenamine in water;

TEa: T0 +Methenamine in acidify water; $p=$ Probability; St: stéatose; Grossissement: 400X.

Figure 3. Histological structure of the kidney of broiler chickens as affected by methenamine feeding regimes.

\section{Discussion}

The inclusion of methenamine in drinking water and feed significantly $(\mathrm{p}<$ 0.05 ) increased feed intake compared to the control groups. The increasing trend in feed intake observed in this study could be attributed to the odourless and sweet taste of methenamine which may have enhanced the palatability of feed. The present result contradicted the findings of Kana et al. [13] who reported that $2 \%, 4 \% \mathrm{D}$. glomerata in feed respectively did not have any significant effect on feed intake of broiler chickens in the starter phase. These authors explained the results by the strong odour and bitter taste of the additives.

Feeding broiler with methenamine through non acidified water significantly ( $\mathrm{p}<0.05$ ) increased body weight and weight gain irrespective of gender. This result could be explained by the high solubility of methenamine in water [7] that facilitates its transport and diffusion or hydrolysis, making available sufficient quantity of formaldehyde that acted on the bacterial flora to increase the quantity of nutrients available to the animal, thus improved weight performance. This result is contrary to that of Della et al. [15] who noted a significant decreased in body weight of rats with the inclusion of $1 \%$ methenamine in drinking water. The difference in this result could be linked to the animal species which changes from one study to another.

The significant decrease in FCR of broiler fed on methenamine through non acidified water could be due to the capacity of water to facilitate the action of methenamine that contributed to the good digestion of feed, availability and better absorption of nutrients leading to the improvement of growth perfor- 
mance [16]. This result agrees with the studies of Basmacioglu et al. [17] who reported the positive effect of feed additives on FCR. Mbajiorgu et al. [18] reported that amino acids used alone or in combination improve FCR and growth performance in broilers.

ANOVA revealed that methenamine incorporated in feed, acidified and non acidified water did not significantly affect carcass yield, liver, pancreas, heart, gizzard, head, legs, abdominal fat and relative weight of digestive organs compared to the negative and positive control treatments. This is in agreement with Ebile et al. [19] who reported similar results by feeding quails with Dichrostachtys glomerata powder in feed and water. In the same line, Gunal et al. [20], Abdel-Fattah et al. [21] and Kana et al. [22] observed no significant effect with chemical and biological feed additives such as antibiotics, organic acids, enzymes and plant charcoal respectively on these parameters.

The number of lactic acid bacteria significantly increased in the digestive tract with the inclusion of methenamine both in feed and in acidified or unacidified water. With the exception of the acidified water, all the other treatments were comparable for the number of coliforms. Despite the total absence of salmonella which can be attributed to limited risk of infection due to the fact that the chickens were housed in cages, the increase in beneficial bacteria at the expense of pathogenic bacteria could be due to the selective antimicrobial action of methenamine that inhibited the growth of pathogens. Methenamine inhibits the proliferation of pathogenic bacteria and modifies the structure of the intestinal epithelium via formaldehyde and ammonia released [11].

Coccidia count decreased in all treatment groups except in broilers fed on methenamine through acidified water during the first two weeks. This suggests that the external supply of acid through water rather inhibited the action of methenamine on coccidia. However, in the third week, coccidia was absent in the positive control group and in the group fed on methenamine through non acidified water. The decreased in the risk of contamination can be due to the fact that the birds were housed in cages. Leni et al. [23] reported that the risk of contamination is lower when birds of the same species are distance from each other. Coccidia were totally absent in all the treatment groups the last week of experimentation. This could be due either to the presence of strengthening of the immune system of the chickens or to the auto-inhibiting action of coccidia. The present result is in agreement with those of Hadi et al. [10] and Leni et al. [23] who respectively mentioned that in avian medicine methenamine acts as an anticoccidial and coccidia has a self-limiting character of development of the disease they caused.

Blood haematological parameters of broilers indicated no significant ( $\mathrm{p}>$ $0.05)$ difference irrespective of the gender and treatment, except the concentration of WBC and PLT that significantly $(\mathrm{p}<0.05)$ decrease in male fed on methenamine compared to the positive control (T0). Feed may have reduced the solubility or hydrolysis of methenamine and therefore its activity in the provi- 
sion of nutrients (proteins, vit A) favorable for the multiplication of white blood cells. This result contradicted the finding of Tesseraud [24] and Olayemi et al. [25] who reported that a protein-rich food increases the level of white blood cells in blood. Likewise, studies by Gbore [26] revealed that the incorporation of monosodium glutamate (GMS) at different rates ( $1 \mathrm{mg}, 2 \mathrm{mg}$ and $4 \mathrm{mg}$ ) in the feed of rabbits significantly increases the average values of white blood cells compared to the control group. However, in males platelet count decreased significantly with the inclusion of methenamine in acidified and non-acidified water while in females it was significantly higher with non-acidified water. The result obtained here could be linked to the increased muscle synthesis activity in males than in females, hence the primary mobilization of feed proteins for this purpose.

ANOVA revealed that serum creatinine level increases significantly when methenamine was administered through acidified water, while urea significantly increased with the administration of methenamine in feed and in non-acidified water. This result suggests that, the acid may have accelerated the degradation of feed creatine or its production by the body muscles, methenamine increased the quantity of protein available, therefore desamination reactions. According to Zounongo [27], the production of urea increases with protein concentration in feed. Analysis of the histological section of the kidneys shows that animals treated through feed and acidified water have a disorganized structure in which the structures of the glomerular were almost indistinguishable and also steatosis characterized by the dilation of the inter glomerular spaces. The variation in creatinine and urea levels in this study did not exceed normal limits, which suggests that methenamine was possibly non-toxic to the kidneys. This agrees with the result of Evgenii and Vladimi [28] who reported that methenamine silver complex has no toxic effects on blood parameters of broilers.

Serum content in total and LDL-cholesterol significantly decreased while HDL-cholesterol increased in broilers fed on methenamine through non acidified drinking water. This suggests that methenamine modulated lipid metabolism to limit the atheromatous risks. This result also suggests that methenamine did not impair liver function and/or limited the amount of fat mobilized by the animals.

\section{Conclusion}

This study revealed that, the administration of methenamine to the broilers through feed, acidified and non acidified water significantly improve growth performance, balance gut microbiota without any negative effect on the liver, kidney and general health of broilers. Methenamine can then be used as feed additive to mitigate the problem of antibioresistance in animal production.

\section{Conflicts of Interest}

The authors declare no conflicts of interest regarding the publication of this paper. 


\section{References}

[1] Kana, J.R., Teguia, A., Mungfu, B.M. and Tchoumboue, J. (2011) Growth Performance and Carcass Characteristics of Broiler Chickens Fed Diets Supplemented with Graded Levels of Charcoal from Maize Cob or Seed of Canarium schweinfurthii Engl. Tropical Animal Health Production, 43, 51-56. https://doi.org/10.1007/s11250-010-9653-8

[2] Ciza, A.P., Kana, J.R., Ngouana, T.R., Nzako, S.A., Kenfack, A., Kemajou, B.Q., Djamen, T.C., Simo, L.P., Kemmo, K.A., Ngouamen, N.T., Dongmo, N.K. and Teguia, A. (2018) Growth Performance, Microbial and Hematological Profile, and Organs Histology of Broiler Chickens Fed with a Seasoning Named jumbo cube as Source of Monosodium Glutamate. Journal of Advanced Veterinary and Animal Research, 5, 146-154. https://doi.org/10.5455/javar.2018.e258

[3] EFSA (2015) Scientific Opinion on the Safety and Efficacy of Hexamethylene Tetramine as a Silage Additive for Pigs, Poultry, Bovines, Sheep, Goats, Rabbits and Horses. EFSA Journal, 13, 4014. https://doi.org/10.2903/j.efsa.2015.4014

[4] Williams, G.J., Hodson, E.H., Isaacs, D. and Craig, J.C. (2012) Diagnosis and Management of Urinary Tract Infection in Children. Journal of Paediatrics and Child Health, 48, 296-301. https://doi.org/10.1111/j.1440-1754.2010.01925.x

[5] Ahrens, F.A. and Martin, R.J. (2013) Antimicrobial Drugs. In: Hsu, W., Ed., Handbook of Veterinary Pharmacology, Blackwell Publishing, Hoboken, New Jersey, 347359.

[6] Al-Badr, A. and Al-Shaikh, G. (2013) Recurrent Urinary Tract Infections Management in Women. A Review. Sultan Qaboos University Medecine Journal, 13, 359. https://doi.org/10.12816/0003256

[7] EFSA (2014) Scientific Opinion on the Re-Evaluation of Hexamethylene Tetramine (E 239) as a Food Additive. The EFSA Journal, 12, 3696. https://doi.org/10.2903/j.efsa.2014.3696

[8] Mohebbi-Fani, M., Shekarforoosh, S., Maleki, M. and Vahedi, N. (2002) Primary Evaluation of Methenamine as a NPN Compound with Probable Effects on Increasing Ruminal Escaped Protein. Journal of Veterinary Medicine Series A, 49, 239-243. https://doi.org/10.1046/j.1439-0442.2002.00446.x

[9] Papich, M.G. (2016) Manuel des medicaments veterinaries. 4th Edition, Elsevier, 505-506. https://doi.org/10.1016/B978-0-323-24485-5.00376-4

[10] Hadi, T.A., Amin, D. and Samaneh, N.G. (2013) Short Preliminary Experimental Study on Teratogenic Effect of Methenamine in Embryonic Model. International Journal of Advanced Biology and Biomedecine Research, 1, 1523-1528.

[11] Afsset (2009) Relatif aux risques sanitaires liés à la présence de formaldéhyde dans les environnements professionnels. Maisons-Alfort, 2004/016, 397 p.

[12] EFSA (2004) Opinion of the Scientific Panel on Additives and Products or Substances Used in Animal Feed on a Request from the Commission on Safety of Formaldehyde for Poultry as Feed Additive in Accordance with Council Directive 70/524/EEC. The EFSA Journal, 96, 1-5. https://doi.org/10.2903/j.efsa.2004.96

[13] Kana, J.R., Mube, K.H., Ngouana, T.R., Tsafong, F., Komguep, R., Yangoue, A. and Teguia, A. (2017) Effect of Dietary Mimosa Small Bell (Dichostachys glomerata) Fruit Supplement as Alternative to Antibiotic Growth Promoter for Broiler Chicken. Journal of World Poultry Research, 7, 27-34.

[14] Ciza Azine, P., Kana, J.R., Ngouana, T.R., Kemmo, K.A., Ngouamen, N.T. and Teguia, A. (2019) Effect of Dietary Supplementation of Graded Levels of Monosodium 
Glutamate (MSG) on Growth Performances, Intestinal Micro Flora, Blood Profile and Organs Histology in Broiler Chickens. International Journal of Livestock Research, 9, 28-40.

[15] Della, P.C., Cabral, I.R. and Parmiani, G. (1970) Transplacental Toxicity and Carcinogenesis Studies in Rats with Hexamethylenetetramine. Tumori Journal, 56, 325-334. https://doi.org/10.1177/030089167005600602

[16] Windisch, W.M., Schedle, K., Plitzner, C. and Kroismayr, A. (2008) Use of Phytogenic Products as Feed Additives for Swine and Poultry. Journal of Animal Science, 86, E140-E148. https://doi.org/10.2527/jas.2007-0459

[17] Basmacioglu Malayoglu, H., Baysal, S., Misirlioglu, Z., Polat, M., Yilmaz, H. and Turan, N. (2010) Effects of Oregano Essential Oil with or without Feed Enzymes on Growth Performance, Digestive Enzyme, Nutrient Digestibility, Lipid Metabolism and Immune Response of Broilers Fed on Wheat-Soybean Meal Diets. British Poultry Science, 51, 67-80. https://doi.org/10.1080/00071660903573702

[18] Mbajiorgu, C.A., Ng'ambi, J.W. and Norris, D. (2007) Effect of Time of Initiation of Feeding after Hatching and Influence of Dietary Lysine Supplementation on Productivity and Carcass Characteristics of Ross 308 Broiler Chickens in South Africa. Livestock Research for Rural Development, 19, Article No. 147. http://www.cipav.org.co/irrd/irrd19/10/mbaj119147.htm

[19] Ebile Dayan, A., Kana, J.R., Pimagha Moffo, H.J., Edie Nounamo, L.W., Nguefack Djieufo, G., Ngouana Tadjong, R. and Fonteh, A.F. (2018) Effects of Dichrostachys glomerata Feeding Regimes on Growth Performance, Gut Microbiota and Haemato-Biochemical Profile of Japanese Quails. Journal of Animal Research and Nutrition, 3, 5 .

[20] Gunal, M., Yayli, G., Kaya, O., Karahan, N. and Sulak, O. (2006) The Effect of Antibiotic Growth Promoter, Probiotic or Organic Acid Supplementation on Performance, Intestinal Microflora and Tissue of Broilers. International Journal of Poultry Science, 5, 149-155. https://doi.org/10.3923/ijps.2006.149.155

[21] Abdel-Fattah, S.A., El-Sanhoury, M.H., El-Mednay, N.M. and Abdel-Azeem, F. (2008) Thyroid Activity, Some Blood Constituents, Organs Morphology and Performance of Broiler Chicks Fed Supplemental Organic Acids. International Journal of Poultry Science, 7, 215-222. https://doi.org/10.3923/ijps.2008.215.222

[22] Kana, J.R. (2010) Le charbon de noyau de fruit noir (Canaruim schweinfurthii Engl) ou de rafle de maïs (Zea mays L.) comme capteur de toxine et activateur de croissance chez le poulet de chair. Thèse de Doctorat/PhD. Faculté d'Agronomie et des sciences Agricole, Université de Dschang, 180 p.

[23] Léni, C. and Guérin, J.L. (2010) Les acaoccidioses aviaire. Ecole national de veterinaire, $6 \mathrm{p}$.

[24] Tesseraud, S. (1995) Métabolisme protéique chez les poulets de chair en croissance. Effet des protéines alimentaires. INRA Production Animales, 8, 197-212. https://doi.org/10.20870/productions-animales.1995.8.3.4128

[25] Olayemi, F., Oyewalo, J., Samson, R. and Olayinka, O. (2003) Comparative Assessment of the White Blood Cells Values, Plasma Volume and Blood Volume in Young and Adult Nigeria Duck. Vetenary Research Programme, 73, 271-276.

[26] Gbore, F.A., Olubu, R.O., Irewole, M.A., Ruth, A.O. and Ajobiewe, G. (2016) Oral Administration of Monosodium Glutamate Alters Growth and Blood Parameters in Female Rabbits. European Journal of Biological Research, 6, 218-225.

[27] Zounongo, M.Z. (2013) Détermination des paramètres biochimiques usuels chez les petits ruminants du Burkin Faso et leurs variations chez les sujets infectés 
naturellement par la trypanosomose. Thèse de Doctorat, Faculté de Médecine, de Pharmacie et d'Odonto-Stomatologie de Dakar, Sénégal, 106 p.

[28] Evgenii, P. and Vladimir, P. (2018) Biological Influence of Methenamine Silver on Broilers. International Journal of Current Pharmaceutical Research, 2, 1.

https://doi.org/10.22159/ijcpr.2018v10i2.25880 